\title{
Plasmaférese como tratamento de glomeruloesclerose segmentar focal (GESF) recorrente após transplante renal. Relato de caso e revisão da literatura Plasmapheresis as a therapy for relapsed focal segmental glomerulosclerosis (FSGS) after kidney transplantation: Case report and a review of the literature
}

Mara C. Moraes

Eduardo G. Roveri ${ }^{1}$

Lívia C. Oliveira

Sumara Z. P. Rigatto ${ }^{2}$

Vera M. S. Belangero ${ }^{2}$

José Francisco C. Marques $J r^{1}$
A glomerulosclerose segmentar focal (GESF) é uma doença renal caracterizada por síndrome nefrótica, com freqüente progressão para insuficiência renal terminal. Nesta fase, o transplante renal, tanto com doador cadáver como intervivos, aparece como a única opção terapêutica para esses pacientes. Contudo, após o transplante renal, a taxa de recorrência de GESF é alta, chegando até $50 \%$ dos casos. Nesta situação, a causa parece estar relacionada a um "fator humoral" circulante, responsável pelo aumento da permeabilidade glomerular. A remoção desse "fator humoral", por meio da plasmaférese terapêutica, aparece como uma boa opção de tratamento para esses pacientes. Neste artigo descreveremos o caso de um paciente masculino de 12 anos de idade, com diagnóstico de glomeruloesclerose segmentar focal recorrente após transplante renal, submetido a tratamento com plasmaférese. Após cinco sessões o paciente evoluiu com melhora sustentada da função renal. O uso de plasmaférese para tratamento da glomeruloesclerose segmentar focal pré e pós-transplante renal ainda é limitado, a maioria dos estudos apresentando resultados de curto prazo ou com pequeno número de pacientes. Alguns fatores preditivos de boa resposta ao tratamento têm sido identificados por diversos autores, dentre eles o início precoce do tratamento após a recorrência e a baixa idade. O número de sessões necessárias para atingir a remissão varia bastante e deve ser determinado individualmente. Outra possibilidade apresentada pelos autores é a plasmaférese profilática, não sendo possível até agora determinar sua eficácia. O caso apresentado é um exemplo de glomeruloesclerose recorrente com ótima resposta a plasmaférese terapêutica, evidenciando o potencial dessa modalidade de tratamento nesta situação e reforçando a necessidade de mais estudos. Rev. bras. hematol. hemoter. 2007;29(2):193-197.

Palavras-chave: Glomeruloesclerose segmentar focal; plasmaférese terapêutica..

\section{Introdução}

A glomeruloesclerose segmentar focal (GESF) é uma doença renal caracterizada por síndrome nefrótica, que acomete tanto as crianças quanto os adultos. Aproximadamente $7 \%-15 \%$ de todas as crianças com síndrome nefrótica idiopática, apresentam diagnóstico de GESF à biópsia renal. Quando não tratados, esses pacientes evoluem com proteinúria progressiva, queda da taxa de filtração glomerular e, em até $30 \%$ dos casos, progridem para insuficiência renal terminal em cerca de vinte anos. ${ }^{1}$

O transplante é o tratamento de escolha para a doença renal terminal. Contudo, o índice de recorrência de GESF é alto, ocorrendo de $30 \%$ a $50 \%$ dos casos. Nesta situação ocorre recorrência da proteinúria com perda do enxerto de 58\% a 80\%, além do risco de recorrência de GESF aumentar muito em transplantes subseqüentes, podendo chegar até a $85 \%$ dos casos.

${ }^{1}$ Centro de Hematologia e Hemoterapia Universidade Estadual de Campinas (Unicamp)

${ }^{2}$ Departamento de Pediatria Universidade Estadual de Campinas.

Correspondência: José Francisco C. Marques Jr

Hemocentro Unicamp - Caixa Postal 6198

13081-970 - Campinas-SP - Brasil

E-mail:marquesj@unicamp.br 
A grande maioria das recorrências é precoce, ocorrendo dentro de três meses do transplante. ${ }^{2-5}$ Casos de remissão espontânea foram observados, porém, são raros. ${ }^{6} \mathrm{~A}$ observação de que na maioria dos casos de recorrência a proteinúria aparece dentro de poucas horas do transplante, sugere a possibilidade de um fator circulante humoral responsável pelo aumento da permeabilidade da membrana basal glomerular, proteinúria e, eventualmente, GESF. ${ }^{7,8}$

Savin et $\mathrm{al}^{7}$ identificaram no plasma de pacientes com recorrência de GESF uma substância que causa aumento imediato da permeabilidade glomerular em camundongos. Estudos posteriores sugerem que este "fator humoral", com aparente massa molecular de aproximadamente $50 \mathrm{Kd}$, seria o responsável pela recorrência de GESF após o transplante renal. $^{7}$

As opções de tratamento medicamentoso para a GESF recorrente são limitadas, visto que a maioria dos pacientes já está em uso de imunossupressão com corticóides, tacrolimus ou ciclosporina, medicamentos utilizados nos casos de GESF idiopática. ${ }^{9}$ A evidência de um possível "fator humoral" torna a plasmaférese uma opção racional de tratamento.

A plasmaférese consiste na remoção do plasma, com retorno das células sangüíneas, separadas por centrifugação, em um circuito extracorpóreo. A grande vantagem deste procedimento está na possibilidade de tratamento de diversas afecções, a partir da remoção de substâncias solúveis no plasma responsáveis pela fisiopatologia de várias doenças, como a púrpura trombocitopênica trombótica, a síndrome de hiperviscosidade a miastenia gravis, entre outras.

Na GESF recorrente, a plasmaférese atuaria removendo o "fator humoral", com conseqüente redução da permeabilidade glomerular e melhora da proteinúria. Teoricamente, atuaria ainda prevenindo a recorrência se usada de forma profilática após transplante renal. Contudo, o uso de plasmaférese para GESF, pré e pós-transplante renal, apresenta resultados limitados, sendo que, na maioria dos estudos até o momento, apresentam resultados de curto prazo ou com pequeno número de pacientes..$^{10-12}$

\section{Relato do Caso}

Paciente LHSA, 12 anos, sexo masculino, estudante, em acompanhamento no serviço de Nefrologia Pediátrica do Hospital das Clínicas da Unicamp desde julho de 1995, com diagnóstico de síndrome nefrótica por glomeruloesclerose segmentar focal, realizado em outro serviço em janeiro do mesmo ano. Ao início do acompanhamento apresentava aos exames uma proteinúria de 2,92 g/24 horas.

Durante a evolução apresentou falha no tratamento com corticosteróides e outros agentes imunossupressores, com aumento da proteinúria e perda progressiva de função renal. Evoluiu ainda com hipertensão arterial e hipogamaglobulinemia secundárias, sendo necessárias várias internações por intercorrências infecciosas ou hipertensão arterial.

Iniciou tratamento dialítico em junho de 2003, quando apresentava proteinúria $8,25 \mathrm{~g} / 24$ horas, creatinina $5,67 \mathrm{mg} / \mathrm{dL}$ e clearence de creatinina $10 \mathrm{~mL} / \mathrm{min} / 1,73 \mathrm{~m}^{2} \mathrm{SC}$.

Foi submetido a transplante renal de doador cadáver em 18/07/2005. A imunossupressão inicial foi realizada com tacrolimus, micofenolato mofetil e basiliximab. Evoluiu com necrose tubular aguda, caracterizada por oligúria e creatinina persistentemente elevada, além de recidiva da GESF, caracterizada por proteinúria crescente, superior a $1 \mathrm{~g} / 24$ horas, sete dias após o transplante. Frente ao quadro de recorrência e com base em trabalhos mostrando resultados promissores foi optado por realizar plasmaférese terapêutica.

Os dados laboratoriais pré e pós-transplante, assim como pré e pós de cada sessão de plasmaférese encontramse detalhados na Tabela 1.

A plasmaférese foi realizada com o equipamento Fresenius modelo AS 104 (Germany), de fluxo contínuo, acoplada ao paciente por meio de cateter central, localizado em veia jugular interna esquerda. Foram realizadas cinco sessões, em dias intercalados, com início em 27/07/2005 e término em $05 / 08 / 2005$, intercaladas com pulso de metilprednisolona $10 \mathrm{mg} / \mathrm{kg} /$ dose. Em cada sessão foi trocada aproximadamen-

Tabela 1: Resultados dos exames de laboratório e correlação com os dias após transplante renal e sessões de plasmaférese

\begin{tabular}{|c|c|c|c|c|c|c|c|c|c|c|c|c|c|c|}
\hline Data (2005) & $17 / 07$ & $18 / 07$ & $19 / 07$ & $22 / 07$ & $23 / 07$ & $25 / 07$ & $27 / 07$ & $29 / 07$ & 01/08 & 03/08 & 05/08 & 09/08 & $12 / 08$ & $16 / 08$ \\
\hline Dias pós-TX & -1 & 0 & +1 & +4 & +5 & +7 & +9 & +11 & +14 & +16 & +18 & +22 & +25 & +28 \\
\hline $\mathrm{Hb} \quad(\mathrm{g} / \mathrm{dl})$ & 12,5 & 9,9 & 8 & 5,6 & 5,7 & 4,5 & 7,8 & 8,8 & 8,4 & 7,8 & 7,4 & & & \\
\hline Leuc $(x 109 / 1)$ & 9730 & 15250 & 15020 & 9680 & 6270 & 14910 & 13440 & 12580 & 13090 & 12020 & 10050 & & & \\
\hline PLT $\left(x 10^{9} / l\right)$ & 262.000 & 255.000 & 272.000 & 194.000 & 222.000 & 303.000 & 314.000 & 348.000 & 324.000 & 348.000 & 397.000 & & & \\
\hline Uréia (mg/dl) & 112 & 111 & 105 & 131 & 180 & 224 & 112 & 151 & 90 & 93 & 89 & 54 & 21 & \\
\hline Creat $(\mathrm{mg} / \mathrm{dl})$ & 9,2 & 8,5 & 6,55 & 7,27 & 8,26 & 10,98 & 6,71 & 7,73 & 3,77 & 2,91 & 1,67 & 1,18 & 0,81 & \\
\hline prot Urina & & & $3+$ & $3+$ & & & $4+$ & $4+$ & & & & NEG & & \\
\hline Ptn (g/24h) & & & & & & 1,26 & & & 0,66 & & 0,07 & & 0,18 & 0,06 \\
\hline Procedimentos & & & & & & & PF & PF & PF & PF & PF & & & \\
\hline
\end{tabular}

OBS: Datas referentes ao ano de 2005; PF: plasmaférese; Ptn: proteinúria; TX: Transplante renal. 


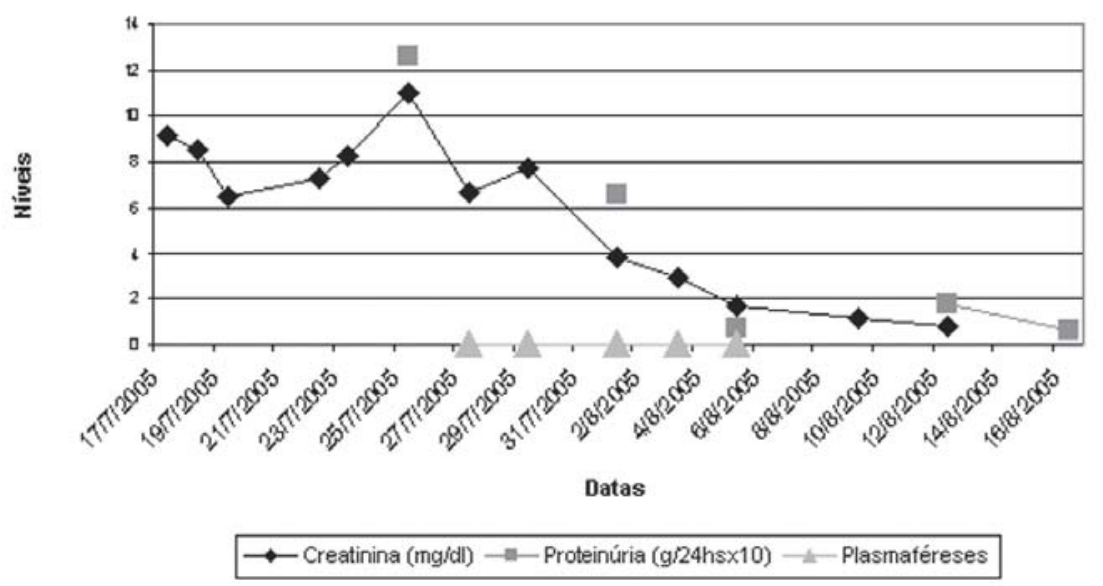

Gráfico 1: Influência das sessões de plasmaféreses em níveis de creatinina e proteinúria

completa, um (8\%) apresentou resposta parcial e três (23\%) apresentaram resposta parcial, porém ficaram dependentes da plasmaférese. Os demais não apresentaram resposta.

Outro fator preditivo de boa resposta parece ser o tempo da recaída até o início do tratamento com plasmaférese. Vários autores ${ }^{14-17}$ observaram que quanto mais precoce é a introdução da plasmaférese após a recaída, melhor é a evolução do paciente, sugerindo que quanto mais cedo o "fator humoral" for retirado da circulação menor é o dano glomerular.

Greenstein et al ${ }^{10}$ evidenciaram que os três pacientes que iniciaram plasmaférese até 48 horas da recaída apresenta-

te uma volemia plasmática, sendo o cálculo da volemia realizado para um valor de hematócrito ideal de $40 \%$ e o peso do paciente de $28 \mathrm{~kg}$. Antes do início do procedimento foi realizada infusão de cloreto de sódio 0,9\% 100 mL, com o objetivo de prevenir a hipotensão. O procedimento foi isovolêmico e como fluido de reposição foi utilizada solução de albumina a 4\%, num volume total de $1000 \mathrm{~mL}$ por sessão, continuamente, durante todo o procedimento.

O paciente não recebeu transfusão de hemocomponentes durante os procedimentos, contudo, nesse período foi necessária a transfusão de um concentrado de hemácias irradiadas (255 mL) no dia 30/07/2005, entre a segunda e a terceira sessões. $\mathrm{O}$ paciente não apresentou qualquer reação ou intercorrência clínica durante ou após os procedimentos.

Como observado na Tabela 1, o paciente evoluiu com melhora progressiva dos níveis de creatinina e redução da proteinúria durante o período submetido a plasmaférese terapêutica, com manutenção dos valores após a suspensão do tratamento. Quatro semanas após o transplante foi introduzido enalapril $5 \mathrm{mg}$ 12/12 horas.

No último acompanhamento, em março de 2006, no dia 209 pós-transplante, o paciente apresentava creatinina 0,99 mg/dL, proteína urinária de 0.05 g/24h e clearence de creatinina de $77 \mathrm{~mL} / \mathrm{min} / 1,73 \mathrm{~m}^{2} \mathrm{SC}$.

No Gráfico 1, evidenciamos a influência das sessões de plasmaféreses nos níveis de creatinina e proteinúria.

\section{Discussão}

O uso de plasmaférese para tratamento de GESF recorrente ainda não está totalmente estabelecido. Em crianças, esse tratamento parece ser efetivo, contudo, o resultado em adultos é mais controverso. Greenstein et al ${ }^{10}$ relataram, em apenas pacientes pediátricos, remissão em cinco de seis (83\%) pacientes tratados com plasmaférese. Diferentemente, Matalon et al $^{13}$ analisaram o resultado do tratamento em 13 pacientes adultos: um paciente (8\%) apresentou resposta ram remissão mais precoce, dentro de 53 dias. Por outro lado, dos dois pacientes que iniciaram tratamento após trinta dias da recaída, um apresentou remissão após 292 dias, enquanto o outro não atingiu remissão.

Pradhan et al ${ }^{16}$ também observaram que quatro de seis pacientes que iniciaram tratamento até um dia de recorrência entraram em remissão até 27 dias, enquanto os dois pacientes que não apresentaram resposta iniciaram o tratamento sete e dezessete dias após o aparecimento da proteinúria.

Em pacientes adultos, o início precoce do tratamento também parece ser um fator importante de resposta como observado por Matalon et al. ${ }^{13}$ Todos os cinco pacientes que apresentaram resposta iniciaram o tratamento em até trinta dias, enquanto sete dos oito pacientes que não apresentaram resposta iniciaram o tratamento após trinta dias da recorrência.

No caso do nosso paciente, o fato de ser criança e ter iniciado a plasmaférese nos primeiros dez dias após a recorrência possivelmente contribuiu para a boa resposta ao tratamento.

Em relação ao número de sessões necessárias para atingir a remissão os dados na literatura são bastante variados. Ponticelli et al $^{18}$ exemplificaram este quadro quando relataram o caso de três pacientes, cada um com uma evolução diferente. Um paciente realizou dois cursos de plasmaférese intensiva, num total de mais de cinqüenta sessões. No último acompanhamento, após dez anos, ainda estava em remissão. Outro paciente permaneceu dependente de plasmaférese para evitar a recorrência e manteve o tratamento por sete anos, quando suspendeu voluntariamente. Após a suspensão evoluiu com síndrome nefrótica e deterioração da função do enxerto. O outro foi um exemplo de ótima resposta ao tratamento, com resposta completa e estável após apenas três sessões. Assim, o número de sessões deve ser determinado individualmente, conforme a evolução clínica do paciente.

A partir desses dados é evidente que quanto mais precoce o início do tratamento, melhor é a evolução e, conse- 
qüentemente, menor o número de sessões necessárias para atingir a remissão. No caso do nosso paciente foram necessárias cinco sessões para que fosse atingida a remissão e, até o momento, no dia 209 após o transplante, não foi observada nova recaída.

A ocorrência de complicações também deve ser considerada quando se avalia a eficácia da plasmaférese como tratamento para GESF recorrente. Como a plasmaférese teoricamente potencializa a imunossupressão, é esperado um aumento do número de infecções ou até de malignidades após o tratamento. Contudo, isto não foi observado pelos diversos autores e, apenas episódios infecciosos isolados foram identificados. ${ }^{10,12}$ Da mesma forma, o nosso paciente evoluiu sem complicações decorrentes do procedimento.

Todos esses resultados promissores levaram alguns autores a testar a eficácia da plasmaférese como profilaxia para a GESF após transplante renal.

Otha et al $^{11}$ analisaram o uso da plasmaférese profilática pré-transplante em 15 crianças com GESF e compararam a resposta ao tratamento de seis pacientes que não realizaram esta profilaxia. Apenas dois dos pacientes que não realizaram profilaxia $(33,3 \%)$ e cinco dos que realizaram $(33,3 \%)$ necessitaram de plasmaférese terapêutica. Somente um dos pacientes sem profilaxia (50\%) respondeu ao tratamento, enquanto todos os cinco que realizaram profilaxia (100\%) apresentaram boa resposta ao tratamento. O nosso paciente não realizou tratamento profilático.

\section{Conclusões}

O caso do nosso paciente foi um exemplo de GESF recorrente de ótima resposta a plasmaférese terapêutica, com evolução semelhante aos casos de melhor resposta descritos na literatura. Assim, frente a esses resultados animadores, o uso da plasmaférese terapêutica para os pacientes com GESF recorrente após transplante renal deve ser considerado, principalmente em pacientes pediátricos e logo no início do aparecimento da proteinúria. Contudo, essa avaliação deve ser feita com cautela, levando em consideração a variedade das respostas observadas. Apenas após novos estudos, prospectivos e randomizados, com um maior número de pacientes, a eficácia desta terapêutica poderá ser confirmada, podendo então ser adotada como rotina.

\section{Abstract}

Focal segmental glomerulosclerosis (FSGS) is a renal disease characterized by a nephrotic syndrome frequently evolving to endstage renal failure. At this stage, renal transplantation, using either cadaver or live donors, is the only therapeutic option. However, after renal transplantation relapse is high, at a rate of $50 \%$ on average. The cause seems to be related to a peripheral humoral factor responsible for increasing glomerular permeability. The clearance of this factor by apheresis is today considered a good therapeutic option. We describe the case of a 12-year-old male patient, with relapsed FSGS after renal transplantation, who was treated by plasmapheresis. After five procedures a sustained improvement in the renal function was obtained. Reports published on plasmapheresis for the treatment of FSGS before and after renal transplantation are still limited to short-term results involving a small number of patients. Some predictive factors for good responses were identified by several investigators including the early start of treatment after relapse and lower ages. The number of plasmapheretic procedures to reach remission varies widely, and should be determined on a case to case basis. Another possibility presented by some investigators is prophylactic plasmapheresis, but this still lacks evidence on efficacy. This case report is an example of FSGS with a good response to plasmapheretic procedures, showing a potential benefit of this treatment. However, further controlled studies involving a higher number of patients are necessary. . Rev. bras. hematol. hemoter. 2007;29(2):193-197.

Key words: Focal segmental glomerulosclerosis; therapeutic apheresis.

\section{Referências Bibliográficas}

1. Wehrmann M, Bohle A, Held H, Schumm G, Kendziorra H, Pressler $\mathrm{H}$. Long-term prognosis of focal sclerosing glomerulonephritis. An analysis of 250 cases with particular regard to tubulointerstitial changes. Clin Nephrol. 1990;33(3):115-22.

2. Datta AR, Sakhuja V, Minz M, Joshi K, Chugh KS. Recurrent focal segmental glomerulosclerosis after renal transplantation. Int J Artif Organs. 1991;14(2):99-101.

3. Artero M, Biava C, Amend W, Tomlanovich S, Vincenti F. Recurrent focal glomerulosclerosis: natural history and response to therapy. Am J Med. 1992;92(4):375-83.

4. Garcia V, Abbud-Filho M, Keitel E, Neumann J, Goldani JC. Recurrent focal glomerulosclerosis in renal allografts. Transplant Proc. 1995;27(1):1084-5.

5. Kim EM, Striegel J, Kim Y, Matas AJ, Najarian JS, Mauer SM. Recurrence of steroid-resistant nephrotic syndrome in kidney transplants is associated with increased acute renal failure and acute rejection. Kidney Int. 1994;45(5):1440-5.

6. Stephanian E, Matas AJ, Mauer SM, Chavers B, Nevins T, Kashtan C, et al. Recurrence of disease in patients retransplanted for focal segmental glomerulosclerosis. Transplantation. 1992; 53 (4):755-7.

7. Savin VJ, Sharma R, Sharma M, McCarthy ET, Swan SK, Ellis E, et al. Circulating factor associated with increased glomerular permeability to albumin in recurrent focal segmental glomerulosclerosis. N Engl J Med. 1996;334(14):878-83.

8. Sharma M, Sharma R, McCarthy ET, Savin VJ. "The FSGS factor:" enrichment and in vivo effect of activity from focal segmental glomerulosclerosis plasma. J Am Soc Nephrol. 1999; 10 (3):552-61.

9. Matalon A, Valeri A, Appel GB. Treatment of focal segmental glomerulosclerosis. Semin Nephrol. 2000;20(3):309-17.

10. Greenstein SM, Delrio M, Ong E, Feuerstein D, Schechner R, Kim D, et al. Plasmapheresis treatment for recurrent focal sclerosis in pediatric renal allografts. Pediatr Nephrol. 2000; 14(12):1061-5.

11. Ohta T, Kawaguchi H, Hattori M, Komatsu Y, Akioka Y, Nagata $\mathrm{M}$, et al. Effect of pre-and postoperative plasmapheresis on posttransplant recurrence of focal segmental glomerulosclerosis in children. Transplantation. 2001;71(5):628-33. 
12. Belson A, Yorgin PD, Al-Uzri AY, Salvatierra O, Higgins J, Alexander SR. Long-term plasmapheresis and protein A column treatment of recurrent FSGS. Pediatr Nephrol. 2001;16(12):985-9.

13. Matalon A, Markowitz GS, Joseph RE, Cohen DJ, Saal SD, Kaplan $\mathrm{B}$, et al. Plasmapheresis treatment of recurrent FSGS in adult renal transplant recipients. Clin Nephrol. 2001;56(4):271-8.

14. Dall'Amico R, Ghiggeri G, Carraro M, Artero M, Ghio L, Zamorani E, et al. Prediction and treatment of recurrent focal segmental glomerulosclerosis after renal transplantation in children. Am J Kidney Dis. 1999;34(6):1048-55.

15. Cheong HI, Han HW, Park HW, Ha IS, Han KS, Lee HS, et al. Early recurrent nephrotic syndrome after renal transplantation in children with focal segmental glomerulosclerosis. Nephrol Dial Transplant. 2000;15(1):78-81.

16. Pradhan M, Petro J, Palmer J, Meyers K, Baluarte HJ. Early use of plasmapheresis for recurrent post-transplant FSGS. Pediatr Nephrol. 2003;18(9):934-8.

17. Dantal J, Baatard R, Hourmant M, Cantarovich D, Buzelin F, Soulillou JP. Recurrent nephrotic syndrome following renal transplantation in patients with focal glomerulosclerosis. A onecenter study of plasma exchange effects. Transplantation. 1991;52 (5):827-31.

18. Ponticelli C, Campise M, Tarantino A. The different patterns of response to plasmapheresis of recurrent focal and segmental glomerulosclerosis. Transplant Proc. 2002;34(8):3069-71.

Avaliação: Editor e dois revisores externos

Conflito de interesse: não declarado

Recebido: 29/06/06

Aceito: 29/12/06 